\title{
Shigella sonnei: ¿Está ocurriendo un cambio en nuestro medio?
}

\author{
Shigella sonnei: is a change occurring in our middle?
}

\author{
José Maria Guevara G ${ }^{1}$, Rosa Cipriani ${ }^{1}$, Dora Giraldo ${ }^{1}$, Elvira Mezarina ${ }^{1}$, Ivonne Sánchez , \\ Zoriada Villagómez ${ }^{1}$, Antonio Antezana ${ }^{1}$, Rosa Alagón', Rosa Carranza ${ }^{1}$ \\ ${ }^{1}$ Instituto de Medicina Tropical, Universidad Nacional Mayor de San Marcos, Lima, Perú.
}

\begin{abstract}
Resumen
Objetivos: Determinar los serogrupos de Shigella como agentes etiológicos de diarrea. Diseño: Estudio descriptivo, retrospectivo, analítico. Institución: Instituto de Medicina Tropical, Universidad Nacional Mayor de San Marcos, Lima, Perú, y Hospital Nacional Daniel A. Carrión, Callao, Perú. Material biológico: Aislamientos de Shigella sp. en coprocultivos. Intervenciones: Se revisó los aislamientos de Shigella sp. en coprocultivos, durante los años 2010 y 2011, en la base de datos WHONET 5.6 del hospital, tanto de pacientes adultos como pediátricos, ambulatorios, hospitalizados y atendidos en emergencia. Se distribuyó la Shigella por serogrupos. Principales medidas de resultados: Frecuencia absoluta y frecuencia relativa de Shigella sp. Resultados: El año 2010 se aisló 64 Shigellas sp.; 41 correspondieron al serogrupo flexneri $(64,1 \%)$ y 20 al serogrupo sonnei $(31,3 \%)$. En el año 2011 se aisló 90 Shigellas sp.; 36 correspondieron a serogrupo flexneri (40\%) y 50 al serogrupo sonnei $(55,6 \%)$. Las diferencias encontradas son estadisticamente significativas. Conclusiones: Shigella sonnei fue el serogrupo predominante en nuestro hospital.
\end{abstract}

Palabras clave: Shigella, coprocultivo, Shigella flexneri, Shigella sonnei.

Abstract

Objectives: To determine Shigella serogroups as diarrhea etiological agents. Design: Descriptive, retrospective, analytical study. Settomg: Instituto de Medicina Tropical, Universidad Nacional Mayor de San Marcos, Lima, Peru, and Hospital Nacional Daniel A. Carrion, Callao, Peru. Biologic material: Shigella sp. isolates in coprocultures. Interventions: Shigella sp. isolates in coprocultures of outpatient, hospitalized and emergency adult and pediatric patients attended during years 2010 and 2011 were reviewed at the hospital WHONET 5.6 database. Shigella were distributed by serogroups. Main outcome measures: Absolute and relative frequency of Shigella sp. Results: Sisty-four Shigellas sp were isolated in 2010; 41 corresponded to flexneri serogroup (64.1\%) and 20 to sonnei serogroup $(31.3 \%)$. Ninety Shigellas sp. were isolated in 2011; 36 corresponded to flexneri serogroup (40\%) and 50 to sonnei serogroup (55,6\%). Differences were statistically significant. Conclusions: Shigella sonnei was the predominant serogroup at our hospital.

Keywords: Shigella, coproculture, Shigella flexneri, Shigella sonnei.

An Fac med. 2014;75(2):189-91 / doi: http://dx.doi.org/10.15381/anales.v75i2.8390

\section{INTRODUCCIÓN}

La Shigella es reconocida por la Organización Mundial de la Salud como un problema mayor de salud pública. Es una de las principales causas de diarrea en pacientes pediátricos en países en desarrollo, mientras que en países desarrollados es causa de diarrea ocasional en niños. Como la mayoría de infecciones gastrointestinales, la shigelosis está asociada con deficiencias en salud ambiental y en la higiene personal ${ }^{(1-4)}$.

La shigelosis es una causa importante de muerte por diarrea; se ha comunicado que no menos de 140 millones de casos de Shigella y 600000 muertes anuales ocurren en el mundo, $60 \%$ de ellos en niños menores de 5 años ${ }^{(5)}$.

Los serogrupos de Shigella están divididos en flexneri, sonnei, boydii y $d y$ senteriae, siendo Shigella sonnei la especie endémica más prevalente en países desarrollados. Shigella flexneri es la más prevalente en países en desarrollo. Shigella dysenteriae es reconocida en producir brotes esporádicos o epidémicos alrededor del mundo ${ }^{(6-10)}$.

El objetivo de nuestro trabajo fue conocer que está sucediendo con la Shigella en nuestro Hospital Nacional Daniel A. Carrión en los últimos años.

\section{MÉTODOS}

El presente es un estudio descriptivo, retrospectivo y analítico. Se revisó en la base de datos WHONET 5.6 del hospital los aislamientos de Shigella sp. en coprocultivos durante los años 2010 y 2011, tanto de pacientes adultos como pediátricos, ambulatorios, hospitalizados y atendidos en emergencia. Se estableció el total de Shigellas aislados en ambos años 2010 y 2011 y también se hizo su distribución por serogrupos.

Para hacer la evaluación nos basamos en la frecuencia absoluta y la frecuencia relativa en los años 2010 y 2011. 
Tabla 1. Aislamientos de Shigella en el Hospital Nacional Daniel A. Carrión 2010-2011.

\begin{tabular}{ccccccc} 
& \multicolumn{2}{c}{2010} & & \multicolumn{2}{c}{2011} \\
\cline { 2 - 3 } \cline { 5 - 6 } Microorganismo & N & $\%$ & & N & $\%$ \\
Shigella boydii & 3 & 4,7 & & 4 & 4,4 \\
Shigella flexneri & 41 & 64,1 & & 36 & 40 \\
Shigella sonnei & 20 & 31,3 & & 50 & 55,6 \\
\hline Total & 64 & 100,0 & & 90 & 100,0 \\
\hline
\end{tabular}

Los datos fueron analizados con Stacalc de Epi Info versión 6. Se realizó la prueba estadística de chi cuadrado con 2 grados de libertad.

\section{RESULTADOS}

En el año 2010 se aisló 64 Shigellas sp. y en el 2011, 90 Shigellas sp. En la tabla 1 y figura 1 se observa la frecuencia y variación de aislamiento de los subgrupos durante estos dos años, y en las tablas 2 y 3, los aislamientos de Shigella por meses. Al comparar la distribución de los serogrupos entre ambos años, se encontró una diferencia estadisticamente significativa $(p=0,01)$.

En la figura 2 se observa los aislamientos de Shigella por trimestre. Se halló que los aislamientos de Shigella sonnei se incrementaron, teniendo un pico entre octubre 2010 y junio 2011.
Asimismo, hubo disminución de la frecuencia de Shigella flexneri en los trimestres observados.

\section{DISCUSIÓN}

La Shigella es un bacilo gran negativo perteneciente a la familia de las enterobacterias. Todos los miembros del género Shigella son patógenos que afectan a humanos, y su efecto de patogenicidad se da en la mucosa gastrointestinal vía la producción de una serie de factores de virulencia ${ }^{(11,12)}$. Es conocido que la circulación de especies y serotipos podría ser considerado como un marcador del clima socioeconómico en un

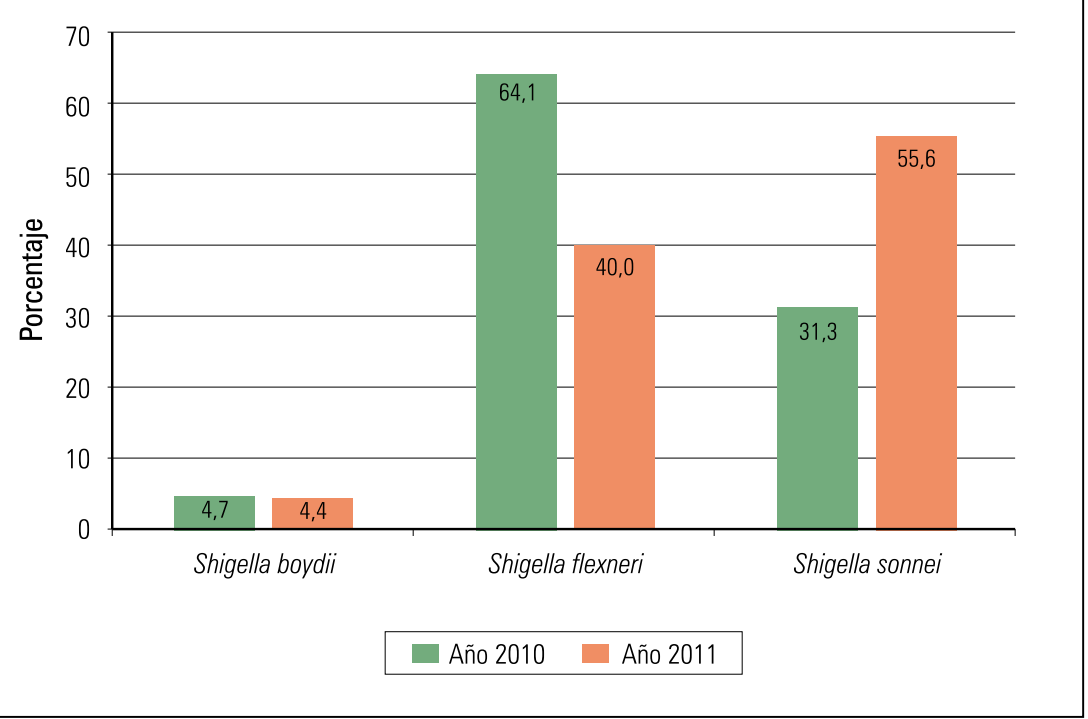

Figura 1. Aislamientos de Shigella en el Hospital Nacional Daniel A. Carrión 2010-2011.

Tabla 2. Aislamientos de Shigella en el 2010, por meses.

\begin{tabular}{ccccccccccccccc} 
Microorganismo & N & $(\%)$ & Enero & Febrero & Marzo & Abril & Mayo & Junio & Julio & Agosto & Setiembre Octubre Noviembre Diciembre \\
Shigella boydii & 3 & 5 & 0 & 1 & 0 & 0 & 2 & 0 & 0 & 0 & 0 & 0 & 0 \\
Shigella flexneri & 41 & 62 & 5 & 5 & 8 & 4 & 3 & 3 & 1 & 1 & 2 & 5 & 0 & 3 \\
Shigella sonnei & 20 & 30 & 0 & 1 & 2 & 2 & 2 & 1 & 2 & 0 & 0 & 1 & 4 \\
Shigella sp. & 2 & 3 & 1 & 0 & 0 & 0 & 0 & 1 & & 0 & 0 & 0 & 0 & 0 \\
\hline Total & 66 & 100 & 6 & 7 & 10 & 6 & 7 & 5 & 3 & 1 & 2 & 6 & 4 \\
\hline
\end{tabular}

Tabla 3. Aislamientos de Shigella en el 2011, por meses.

\begin{tabular}{ccccccccccccccc} 
Microorganismo & $\mathrm{N}$ & $(\%)$ & Enero & Febrero & Marzo & Abril & Mayo & Junio & Julio & Agosto & Setiembre Octubre Noviembre Diciembre \\
Shigella boydii & 4 & 4 & 1 & 1 & 0 & 1 & 0 & 0 & 0 & 0 & 1 & 0 & 0 & 0 \\
Shigella flexneri & 36 & 40 & 2 & 5 & 2 & 0 & 3 & 8 & 2 & 1 & 4 & 1 & 4 & 3 \\
Shigella sonnei & 50 & 56 & 8 & 17 & 5 & 0 & 1 & 2 & 1 & 2 & 1 & 1 & 6 & 6 \\
\hline Total & 90 & 100 & 11 & 23 & 7 & 1 & 4 & 10 & 3 & 3 & 6 & 2 & 10 & 9
\end{tabular}




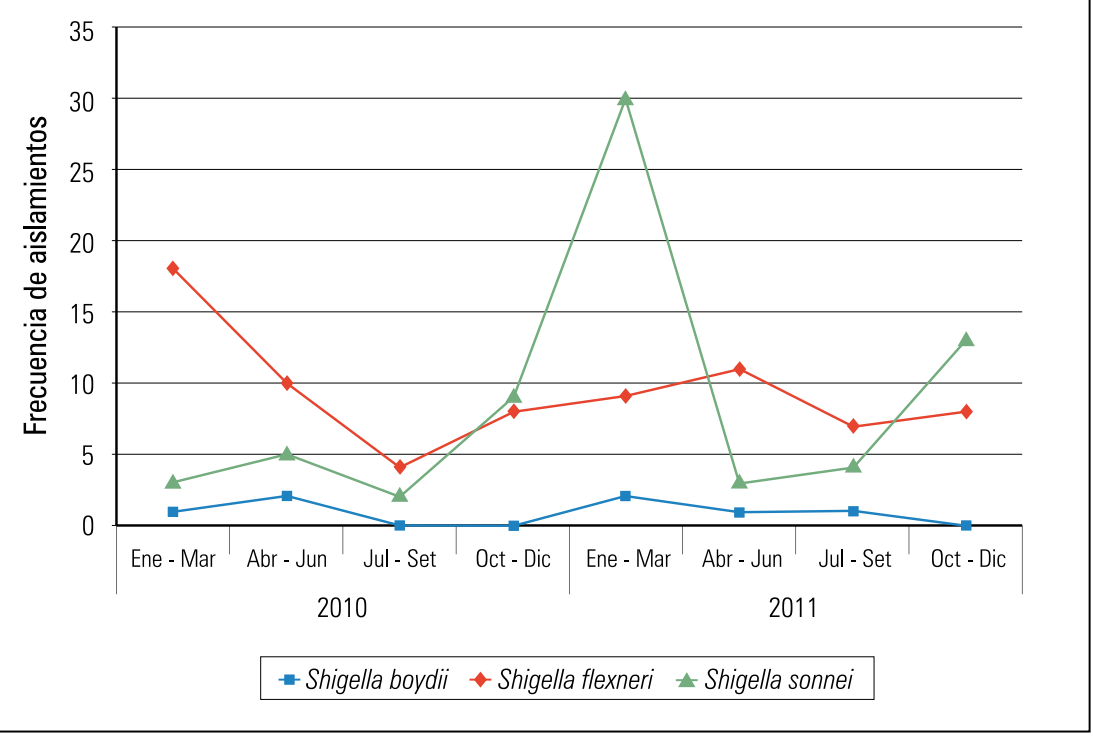

Figura 2. Aislamientos de Shigella por trimestres.

momento dado ${ }^{(13)}$, situación que se ha dado en nuestro hospital, donde en 2011 se incrementó los casos de Shigella sonnei que desplazó a la Shigella flexneri. Este incremento fue significativo comparado con el año anterior. Esto podría estar ocurriendo por diversos factores, que deberían ser estudiados, como el calentamiento global que está padeciendo el mundo, que se demuestra por el aislamiento de esta bacteria ya durante todo el año y la bonanza económica que vive nuestro país. A pesar que la cantidad no es significativa, se tendría que corroborar nuestros resultados con los de otros centros del país.

\section{REFERENCIAS BIBLIOGRÁFICAS}

1. Ferreccio C, Prado V, Ojeda A, Cayyazo M, Abrego $P$, Guers L, Levine MM. Epidemiologic patterns of acute diarrhea and endemic Shigella infections in children in a poor periurban setting in Santiago, Chile. Am J Epidemiol. 1991;134:614-27.

2. Houang ETS, Chu Y-W, Ng T-K, Cheng FB. Study of the relatedness of isolates Shigella flexneri and Shigella sonnei obtained in 1986 and 1987 and in 1994 and 1995 from Hong Kong. J Clin Microbiol. 1998;36:2404-7.

3. Prado V, Pidal P, Arellano C, Lagos R, San Martín O, Levine M. Antimicrobial multiresistence of Shigella spp. strains in a semi rural community of north Santiago. Rev Méd Chile. 1998;126:1464-71.

4. Bennish M, Wojtyniak B. Mortality due to shigellosis: community and hospital data. Rev Infect Dis. 1991;13:S245-S251. doi: http://dx.doi.org/10.1093/ clinids/13.Supplement_4.S245

5. Sur D, Ramamurthy T, Deen J, Bathacharya SK. Shigellosis: challenges \& management issues. Indian J Med Res. 2004;129(5):454-62.
6. Kotloff KL, Winickoff JP, Ivanoff B, Clemens JD, Swerdlow DL, Sansonetti PJ, Adak GK, Levine MM. Global burden of Shigella infections: implications for vaccine development and implementation of control strategies. Bull World Health Organ. 1999;77:651-66.

7. Gupta A, Polyak CS, Bishop RD, Sobel J, Mintz ED. Laboratory-confirmed shigellosis in the United States, 1989-2002: epidemiologic trends and patterns. Clin Infect Dis. 2004;38:1372-7. doi: http://dx.doi. org/10.1086/386326

8. Levine MM, Kotloff KL, Barry EM, Pasetti MF, Sztein MB. Clinical trials of Shigella vaccines: two steps forward and one step back on a long, hard road. Nat Rev Microbiol. 2007;5:540-53. doi: http://dx.doi. org/10.1038/nrmicro1662

9. Ram PK, Crump JA, Gupta SK, Miller MA, Mintz ED. Part II. Analysis of data gaps pertaining to Shigella infections in low and medium human development index countries, 1984-2005. Epidemiol Infect. 2008;136:577-603. doi: http://dx.doi.org/10.1017/ S0950268807009351

10. Toro CS, Farfan M, Contreras I, Flores O, Navarro N, Mora GC, Prado V. Genetic analysis of antibiotic resistance determinants in multi-drug-resistant Shigella strains isolated from Chilean children. Epidemiol Infect. 2005;133:81-6. doi: http://dx.doi. org/10.1017/S0950268804003048

11. Niebuhr K, Sansonetti PJ. Invasion of epithelial cells by bacterial pathogens the paradigm of Shigella. Subcell Biochem. 2000;33:251-87. doi: http:// dx.doi.org/10.1007/978-1-4757-4580-1_11

12. Chompook P, Samosornsuk S, von Seidlein L, Jitsanguansuk S, Sirima N, Sudjai S, Mangjit P, Kim DR, Wheeler JG, Todd J, et al. Estimating the burden of shigellosis in Thailand: 36-month population based surveillance study. Bull World Health Organ. 2005;83(10):739-46.

13. Philpott DJ, Edgeworth JD, Sansonetti PJ. The pathogenesis of Shigella flexneri infection: lessons from in vitro and in vivo studies. Philos Trans R Soc Lond Biol Sci. 2000;355(1397):575-86. doi: http:// dx.doi.org/10.1098/rstb.2000.0599

Financiamiento: Recursos propios.

No existen conflictos de intereses.

Correspondencia:

Dr. José María Guevara Granados

Instituto de Medicina Tropical Daniel A. Carrión, UNMSM, sección Micología

Dirección: José Santos Chocano 199

Correo electrónico: josemaguevaragra@hotmail.com 